\title{
Towards verifying contract regulated service composition
}

\author{
Alessio Lomuscio · Hongyang Qu • Monika Solanki
}

Received: date / Accepted: date

\begin{abstract}
We report on a novel approach to (semi-)automatically compile and verify contractregulated service compositions implemented as multi-agent systems. We model web service behaviours and the contracts governing them as WSBPEL specification. We use the formalism of temporal-epistemic logic, suitably extended to deal with compliance/violations of contracts, to specify properties of service compositions. We compile the WSBPEL behaviours into a specialised system description language ISPL, to be used with the model checker MCMAS to verify the behaviours automatically. We illustrate these concepts using a motivating example whose state space is approximately $10^{6}$ and discuss experimental results.
\end{abstract}

Keywords Multi-agent systems $\cdot$ Web services $\cdot$ Model checking

\section{Introduction}

Web services (WS) are now considered one of the key technologies for building new generations of digital business systems. While a system made of few and localised services may only interact in a small number of ways, when several subsystems are able to coordinate in an open environment the end result may be much less predictable. Certain components may fail, others may be incapacitated to provide the services in the expected timeline and others still may have to adopt a policy of prioritisation among the requests that are being received. Services participating in such compositions therefore need the autonomy to act rationally and maximise their own design objectives while keeping the goals of the composition in perspective [1]. The paradigm of multi-agent systems (MAS) provides a useful

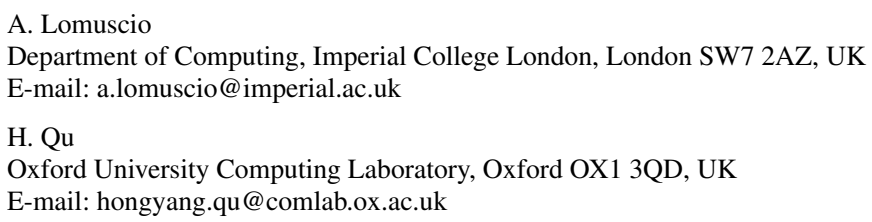


framework for reasoning about services and their compositions. Indeed the $\mathrm{W} 3 \mathrm{C}$ consortium [2] suggested that "A web service is an abstract notion that must be implemented by a concrete agent. The agent is the concrete piece of software or hardware that sends and receives messages."

When services are combined, a significant challenge is to regulate the business interactions between them. While the designer of the system as a whole cannot guarantee an ideal outcome for the service composition - since he or she has no overall control over it - he may still wish to establish verifiable mechanisms to create an incentive in the agents to carry out transactions in a way that is more likely to create an overall positive outcome. One such mechanism is service level agreements (SLAs). SLAs are rules representing agreed level of service provision to be supplied by the agents when interactions are invoked within certain parameters. For example, a certain SLA may prescribe that all requests from a client are to be answered within $1 \mathrm{sec}$ whenever they are requested from 9am to $5 \mathrm{pm}$ and within $3 \mathrm{secs}$ from $5 \mathrm{pm}$ to $9 \mathrm{am}$. While monitoring and identifying responsibilities in the violation of SLAs is a non-trivial problem whenever service inter-dependencies exist, it is well recognised that SLAs can act as a basic regulatory mechanism and may help engineers in predicting the average behaviour of the system. There certainly is an increasing emphasis on reasoning about SLAs in software engineering and in the implementation of platforms supporting them [3, 4].

Although SLAs are useful, they can represent only basic agreements of service provision. But applications running complex, human-like activities require more general and sophisticated declarative specifications certifying legal-like agreements among the parties. These mechanisms should not only describe the intended timeline for the provision of services but would also have to specify obligations, permissions of states and/or actions in a variety of functioning circumstances including those coming into force as a result of certain agents not performing "as expected". A useful concept from the legal domain in this sense is the one of contract as found in human societies. In an environment where previously unknown services are dynamically discovered and bound, their composition may usefully be underpinned by binding agreements or "contracts". Should a contract be broken by one of the parties, legal remedies may be applicable in the form of penalties, additional rights to some party, and, possibly, additional penalties with respect to third parties. A key characteristic of contracts is that they may still be broken. In this setting system engineers may be interested in investigating what behaviours the MAS implements when all agents are fulfilling their contracts, but also, and perhaps more importantly, what properties the system exhibits when some of the agents are violating their contracts in certain ways. Particularly, one may want to check if some really unwanted behaviours may result following certain violations or whether the system provides certain elements of resilience irrespective of agents breaking their contracts. Verification of WS is an active topic of research (e.g., see [5,6]). So far efforts have focused on checking safety and liveness properties only. However when WS are phrased as a contract-regulated MAS there are other properties that seem worth studying, such as various notions of correctness/violations of the contracts during a run, the evolution of the agents' knowledge about themselves, the contracts and the expected peers' behaviours, etc. Indeed, there is a long and successful tradition in the MAS community to use rich logic-based languages to specify the behaviour of agents in the system. In particular, not only is temporal logic used but also, among others, epistemic (to reason about knowledge of the processes), deontic (to reason about obligation of the processes), cooperation (to reason about strategies of the agents), and other modalities. Recent developments in the verification of MAS via model checking techniques [7-9] permit the verification of not only 
plain temporal languages but also a variety of modalities describing the informational states, typically knowledge, of the agents.

In this paper, we propose a novel approach towards the verification of agent based services, where transactions are regulated by binding electronic contracts. In our approach, all possible behaviours of agent based services or "contract parties" (CP) are specified using WSBPEL (BPEL for short) [10]. The contractually correct behaviours for every agent are also specified in BPEL. A compiler is implemented that takes as input both these behaviour descriptions, and generates a model in multi-agent systems as an ISPL program, which is fed to the symbolic model checker MCMAS. By querying MCMAS, we can then verify whether or not contracts may be broken by the agents implementing Web services, and if so, what are the consequences of this.

The rest of the paper is organised as follows. In Section 2 we introduce the trace-based semantics of interpreted systems as the underlying semantics for MAS. In Section 3 we discuss various notions of compliance and violations with respect to contracts. Section 4 presents our proposed verification framework and a brief introduction to BPEL, MCMAS, and ISPL. Section 5 discusses the implementation of the compiler, the key component in the framework, which translates BPEL specifications into ISPL models. Section 6 introduces a motivating example with some typical obligations and violations of contract parties, and presents experimental results of our approach on that example. We conclude in Section 7 , where we also discuss related work. The paper extends the results originally presented in [11, 12].

\section{Temporal deontic interpreted systems}

We introduce here a formalism to express notions pertaining to the temporal evolution of MAS, the knowledge of agents in the system as well as the correctness and violations of states and runs with respect to a predetermined set of contracts regulating the interaction among the agents.

\subsection{Semantics}

We model a MAS as composed of a set of agents and an environment. We assume that each agent is implementing a contract-regulated web service providing particular functionalities. We follow the interpreted system model [13] and assume that at any given time each agent in the system is in a particular local state. This local state can be a state of compliance with respect to the agent's contracts or of violation. Following [14], we will call the former allowed (or green) state, and disallowed (or red) the latter. This is a coarse distinction that can be refined (see [14]), but it suffices for the scope of this paper.

Each agent has a repertoire of actions available; the action selection mechanism is given by the notion of local protocol, effectively a function giving the set of possible actions that may be performed when in a given local state. The system evolves by means of transitions from a collection of (instantaneous) local states to another following the execution of actions for all the agents in the system.

The notions of entitlement, penalty, etc., given by compliance with respect to a given set of contracts are incorporated in the notions of protocol and transition. This will be exemplified in the example of Section 6.1. 
For the above purposes we adopt the model of deontic interpreted systems [14] as extended to temporal models as in [15]. Formally, we assume a set of agents $A=\{1, \ldots, n\}$ and an environment $e$.

To each agent $i$ we associate a set of instantaneous local states $L_{i}$ and a set $L_{e}$ to the environment. For each agent $i$ we assume the set of local states $L_{i}=G_{i} \cup R_{i}$ is partitioned into two subsets: $G_{i}$ represents green (or ideal) local states, $R_{i}$ represents the red (or nonideal). Intuitively $G_{i}$ represents states of compliance with respect to the contracts the agent $i$ is subjected to, whereas $R_{i}$ represents states of violation.

To represent the instantaneous configuration of the whole MAS at a given time we use the notion of global state. A global state $s \in S$ is a tuple $s=\left(l_{1}, \ldots, l_{n}, l_{e}\right)$ where each component $l_{i} \in L_{i}$ represents the local state an agent $i$ is in (these may be either a green or a red state), together with the environment state. The set of all global states $S \subseteq L_{1} \times \cdots \times$ $L_{n} \times L_{e}$ is a subset of the Cartesian product of all local states and the local states for the environment. $I \subseteq S$ is a set of initial states for the system.

The formal model we use accounts for the temporal evolution of the system. To do this we assume $A c t_{i}$ to be the set of actions available to agent $i$ and similarly $A_{c t}$ to the environment. It is assumed null $\in A c t_{i}$ for each agent $i$ where null is the null action. Actions are selected by means of action selection mechanisms local to the agents; this is formalised by local protocol functions $P_{i}: L_{i} \rightarrow 2^{A c t_{i}}$ for any $i \in A$. In other words $P_{i}\left(l_{i}\right)$ represents the actions that may be performed in the state $l_{i}$ (irrespectively as to whether $l_{i}$ is a red or green state). Some of these actions will lead to green states for the agent, others to red ones. A tuple $\left(a_{1}, \ldots, a_{n}, a_{e}\right)$ in which every component represents the action carried out by an agent (the environment for the last component) is called a joint action.

The evolution of the system is given by locked transitions for all the agents and the environment. The model assumes that each agent moves from one local state to another local state at each time tick. The transitions between local states depend on which actions have been performed by all agents in the system. So an agent's action may affect a different agent's resulting next state. Although this is not enforced in the semantics, in any concrete example we will impose that the colour of the resulting target state will be green or red depending on the local action the agent performed. In other words other agents' actions do not have an impact on the colour of the target state; if necessary we can have two copies for a certain local state, one green and one red, to differentiate outcomes depending on the agent's latest action and the ones of the rest of the system. Formally, for each agent we assume a local transition function $\tau_{i}: L_{i} \times A c t_{1} \times \ldots \times A c t_{n} \times A c t_{e} \rightarrow L_{i}$ defining the local state for agent $i$ resulting from a local state and and a joint action.

Local transitions are combined together (the model checker presented later will do precisely this) to give a joint transition function $\tau: S \times A t_{1} \times \ldots \times A_{c t} \times A_{n} t_{e} \rightarrow S$, i.e., $\tau=\tau_{1} \times \ldots \times \tau_{n} \times \tau_{e}$, giving the overall transition function for the system. We write $\left(s, s^{\prime}\right) \in T$ if $\tau\left(s, a_{1}, \ldots, a_{n}, a_{e}\right)=s^{\prime}$ for some joint action $\left(a_{1}, \ldots, a_{n}, a_{e}\right)$.

We introduce paths as standard to give an interpretation to a branching time language. A path $\pi=\left(s_{0}, s_{1}, \ldots, s_{j}\right)$ is a sequence of possible global states such that $\left(s_{i}, s_{i+1}\right) \in T$ for each $0 \leq i<j$. For a path $\pi=\left(s_{0}, s_{1}, \ldots\right)$, we take $\pi(k)=s_{k}$.

Definition 1 (Models) A model $M=\left(S, I, T, \sim_{1}, \ldots, \sim_{n}, h\right)$ is a tuple such that:

- $S \subseteq L_{1} \times, \ldots \times L_{n} \times L_{e}$ is the set of global states for the system,

$-I \subseteq S$ is a set of initial states for the system,

- $T$ is the temporal relation for the system defined as above,

- For each agent $i \sim_{i}$ is an epistemic indistinguishably relation defined by $\left(l_{1}, \ldots, l_{n}, l_{e}\right) \sim_{i}$ $\left(l_{1}^{\prime}, \ldots, l_{n}^{\prime}, l_{e}^{\prime}\right)$ if $l_{i}=l_{i}^{\prime}$. 
- $h: \mathcal{P} \rightarrow 2^{S}$ is an interpretation for a set of propositional atoms $\mathcal{P}$.

The above models allow us to interpret a temporal epistemic language. The relation $\mathrm{T}$ will be used to interpret temporal operators whereas $\sim_{i}$ will be used to interpret epistemic modalities as standard [13].

\subsection{Syntax}

Our formal language is a multi-modal logic including operators for branching time, epistemic operators and specialised local variables expressing correctness and violations. We will see that by combining local propositions for violations and correctness with temporal and epistemic operators we can express a variety of notions of compliance.

Definition 2 (Syntax) The syntax of the specification language is given by the following BNF syntax:

$$
\phi::=p\left|g_{i}(i \in A)\right| \neg \phi|\phi \wedge \phi| K_{i} \phi|E X \phi| E \phi U \phi \mid E G \phi
$$

In the above definition $p$ is an atomic proposition belonging to a set $\mathcal{P}$ that includes the constants true and false. $g_{i}$ is an $i$-local atomic proposition expressing that "agent $i$ is presently in compliance (with respect to a set of contracts)". We use $g_{i}$ as we will often say that in this case "agent $i$ is in a green state". We sometimes write $r_{i}$ for $\neg g_{i}$ expressing that "agent $i$ is presently not in compliance", or "agent $i$ is in a red state". The formula $E X \phi$ stands for "there exists a path accessible from the present state in which $\phi$ holds at the next state"; $E \phi U \psi$ stands for "there exists at least one path where $\psi$ holds at some point in the future and $\phi$ holds in all states until then"; $E G \phi$ stands for "there exists a path accessible from the present state in which $\phi$ always holds at all future states"; $K_{i} \phi$ represents that "agent $i$ knows $\phi$ ". For examples and interpretation of the temporal epistemic fragment we refer to specialised literature on the subject [13].

We can now interpret our logical language. In this paper we are only interested in those states that can be reached from initial states when considering satisfaction of logic formulae. Hence the set of states $S$ in a model $M$ is restricted to reachable states.

Definition 3 (Satisfaction) Satisfaction for a formula $\phi$ in a model $M$ at a global state $s=\left(l_{1}, \ldots, l_{n}, l_{e}\right)$, denoted as $(M, s) \models \phi$, is defined recursively as follows:

- $(M, s) \models$ true;

- $(M, s) \models p$ if $s \in h(p)$;

- $(M, s) \models g_{i}$ if $l_{i} \in G_{i}$;

- $(M, s) \models \neg \phi$ if $(M, s) \not \models \phi$;

- $(M, s) \models \phi \wedge \psi$ if $(M, s) \models \phi$ and $(M, s) \models \psi$;

- $(M, s) \models K_{i} \phi$ if for all $s^{\prime} \in S$ if $s \sim_{i} s^{\prime}$, then $\left(M, s^{\prime}\right) \models \phi$.

- $(M, s) \models E X \phi$ if there exists a path $\pi$ starting at $s$ such that $(M, \pi(1)) \models \phi$;

- $(M, s) \models E \phi U \psi$ if there exists a path $\pi$ starting at $s$ such that for some $k \geq 0$ $(M, \pi(k)) \models \psi$ and $(M, \pi(j)) \models \phi$ for all $0 \leq j<k$;

- $(M, s) \mid=E G \phi$ if there exists a path $\pi$ starting at $s$ such that $(M, \pi(k)) \models \phi$ for all $k \geq 0$

The other connectives $A X, A U, A G, E F, A F$ are defined via the above as standard [16]. For example, $E F \phi=E(\operatorname{true} U \phi)$. Observe that the satisfaction condition for the epistemic 
modality depends on the local states of the agents and therefore the epistemic properties of the system can be verified automatically.

The definition of satisfaction above is customary in temporal epistemic logic and only extends the literature by adding propositional constants $g_{i}$ for compliance of agent $i$. The approach used here is essentially the one presented in [17] to which time is added.

Often we are interested in establishing whether a model $M$ representing a whole system satisfies a specification $\phi$, represented as $M \models \phi$. In this case we will check whether $(M, s) \models \phi$ for all $s \in I$.

By using the syntax above, intuitively we can express specifications representing properties in contract-regulated WS-compositions. For example, we may wish to reason about:

- what properties are brought about by a run of the system in which no agent violates any of his contracts,

- what properties hold true if some of the agents violate (part of) their contracts,

- what knowledge the agents have about the consequences of some other agents violating some of their contracts, and how this knowledge evolves over time.

These can serve as an intuition. The next section will provide further discussion on the expressivity of the language.

\section{Expressivity}

We now formalise various notions of behavioural compliance with respect to a set of contracts. In the following, we abstract from particular contracts the agents are subjected to and instead present a general notion of compliance/violation. There are many dimensions of possible investigation here: compliance may be local or global, it may hold for portions or for the total length of a path, etc. We only focus on abstract notions here, and refer to Section 6 to discuss the applicability of these in concrete scenarios.

The local behaviours of an agent may be classified distinctly into compliant and noncompliant respectively. In crude terms, a compliant behaviour is one in which the agent is always in a green state, whereas a non-compliant one results in some red states being visited.

\subsection{Absolute compliance}

We begin by analysing the notion of compliance of the local behaviour of an agent $i$ over full paths. In particular, we can distinguish between possible local compliance over a path ("there exists a path in which agent $i$ is always in a green state") and a notion of full local compliance ("in all paths agent $i$ will always be in compliance"). The former can be expressed using the syntax in Section 2 by

$$
E G g_{i}
$$

and the latter by

$$
A G g_{i} .
$$

The above allows us to specify easily the consequence of local compliance over full paths for agents. For instance, if we need to express that "whenever agent $i$ is in compliance the state of affairs $\phi$ holds in the system," we could state:

$$
A G\left(g_{i} \rightarrow \phi\right)
$$


Observe the above is a kind of Anderson-Kanger reduction [18,19].

Should we need to refer to states resulting from more than one agent being in compliance we can obviously take the conjunction of the respective $g_{i}$. For instance

$$
A G\left(\bigwedge_{i \in A^{\prime}} g_{i} \rightarrow \phi\right)
$$

represents the fact that $\phi$ holds true whenever all agents in $A^{\prime} \subseteq A$ are in compliance.

By allowing $A^{\prime}$ to grow to represent the entire set of agents we can refer to "full global compliance"

$$
A G\left(\bigwedge_{i \in A} g_{i} \rightarrow \phi\right)
$$

representing "whenever all agents in the system are in compliance $\phi$ holds."

We can combine the above with knowledge modalities. For example we may want to express that an agent $i$ knows that as long as agent $j$ is in compliance a certain state of affairs is always reachable in some way. This may be expressed by

$$
K_{i}\left(A G\left(g_{j} \rightarrow E X \phi\right)\right)
$$

Obviously more complex specifications are expressible and, indeed, useful.

\subsection{Consequences of violations}

In addition to the above, we may be interested in what consequences arise should one agent not be in compliance. For instance we may wish to express that following a violation by agent $i$ a certain state of affairs holds indefinitely and that all other agents know this. This is expressible by the formula

$$
A G\left(\neg g_{i} \rightarrow A G \phi\right) \wedge \bigwedge_{j \neq i} K_{j}\left(A G\left(\neg g_{i} \rightarrow A G \phi\right)\right) .
$$

This may be useful to represent the knowledge the agents have with respect to the consequences of one agent defaulting on his obligations.

Often we are interested in the notion of "recovery". Following a local violation, perhaps there is a way in the system for the agent to recover. Recovery is here simply represented as a transition an agent makes from a red state to a green state. A possible local recovery is expressible in the language defined in Section 2 as

$$
A G\left(\neg g_{i} \rightarrow E F g_{i}\right)
$$

In the language above we also can easily express that all agents always know this,

$$
A G\left(\bigwedge_{i \in A} K_{i} A G\left(\neg g_{i} \rightarrow E F g_{i}\right)\right)
$$

Observe that the simple red/green labelling only offers rather coarse expressivity. In general a contract may have several clauses. In some circumstances an agent could be in violation of one clause only, while in others of several or all clauses: the formalism above cannot distinguish situations of this kind. For this reason, we cannot express recovery with respect to a particular clause. As noted earlier it is possible to extend the deontic interpreted formalisms with further labelling to allow for this; for simplicity we do not do this here. 
Similar formulas may be introduced describing recovery for global violations as well. The reader will fill in these details easily.

Assuming an agent begins a run in a green state, there are at least four classes of evolution that are of interest as shown in Figure 1.

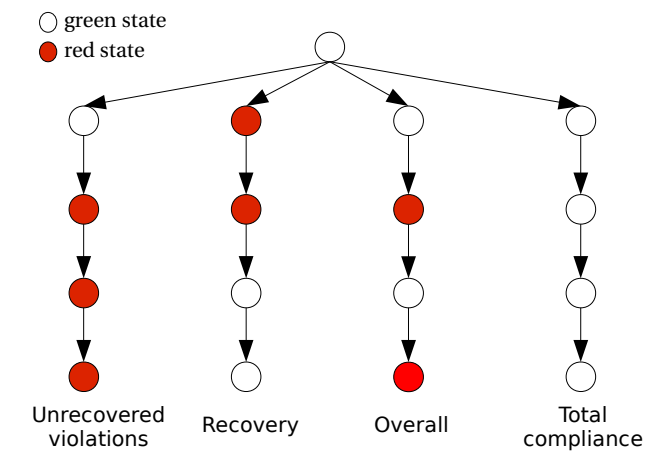

Fig. 1 Various evolutions from an initial green state.

- Overall behaviours: These are behaviours which are represented by paths that have both red and green states in no particular order. These behaviours specify the general, overall behaviour for an agent.

- Totally compliant behaviours: These are behaviours represented by paths covering only green states. The transitions occur when the observed behaviour is in compliance with the contract-compliant behaviour. If a model includes such a path, it satisfies the specification $E G g_{i}$.

- Unrecovered violations behaviours: These are behaviours represented by paths where all states in a given prefix are green, but whose suffix are red. Behaviours represented by such transitions represent non-recoverable behaviours from a violation. The formula $E\left(g_{i} U E G \neg g_{i}\right)$ is true in models that include these paths.

- Recovery behaviours: These are behaviours represented by paths where all states in some suffix of the path are green, but some states in its prefix are red. The formula $E\left(g_{i} U\left(\neg g_{i} \wedge E F E G g_{i}\right)\right)$ is true in models containing at least one of these paths.

But other possibilities exist. For example, we may wish to reason in terms of how many times an agent is in a violation state in a path. The case of two violations may be expressed as:

$$
E\left(g_{i} U\left(\neg g_{i} \wedge E X E\left(g_{i} U\left(\neg g_{i} \wedge E X E G g_{i}\right)\right)\right)\right) .
$$

We present a realistic WS scenario in Section 6 and analyse it in view of the above.

\section{Verification framework}

In this section we present the framework, illustrated in Figure 2, here adopted for the verification of contract regulated MAS implementing web services. For the sake of clarity, the figure only refers to the methodology for agent $C_{1}$. In the actual toolkit similar mechanisms are replicated for all the agents in the composition. 
The architecture proposed takes two inputs for each agent: the set of all arbitrary behaviours $(A B s)$, and the set of contractually compliant behaviours $(C C B S)$. Both sets can in principle be given by means of any transition based structure or program defining it. ABs denote the set of transitions that the agent, or the service, can in principle engage in irrespective of the contracts it has signed up to. For instance, a service may in principle fail to perform certain actions even under the obligation of doing so, or perform actions in violation with certain clauses in specific contracts pertaining to its behaviours. In contrast CCBs denote the set of transitions that arise when the agent acts without deviating from the contracts in question. Note both sets are in principle non-deterministic and in general involve an element of synchronisation with the other agents' actions.

We should wish to be able to consider CCBs as defined by an ad-hoc contract language such as $[20,21]$ that can be interleaved with ABs taken directly from the agent's code. However, interfacing a contract based language to a description of all possible behaviours of an agent is an unresolved problem beyond the scope of this paper. Instead, in line with our current interests in concrete applications, we assume to have both $\mathrm{ABs}$ and $\mathrm{CCBs}$ to be given in BPEL [10], the leading language for the implementation of web services.

In our experience most industrial use cases involving contracts are currently analysed by considering the contract compliant scenarios, i.e., by investigating the CCBs traces. These are often very well understood and described with a range of formal or semi-formal machinery, including transition systems, state charts, and automata. ABs are in some instances poorly analysed and, indeed, it is often because of this, that unwanted outcomes are reached in the service composition. Still, even in the worst case where ABs are not documented in any semi-formal form, ultimately they can be reconstructed from the actual code run by the service. This typically abstracts from the contracts themselves; i.e., the clauses are not represented explicitly in the code, but the agent's code implicitly governs the circumstances in which the agent follows the contracts or otherwise.

As we describe in the next section in more detail, by using ABs and CCBs in conjunction we can colour the overall resulting model and verify the agents' behaviours against their contract-compliant runs. Specifically, we wish to be able to analyse:

- the extent to which an individual agent's behaviour follows the contracts it is supposed to adhere to, and, if not, the extent to which recovery actions are executed by the agent as well as their outcome.

- the extent to which the overall system achieves the intended outcomes of the composition when all, or certain contracts are followed. In case of failure we wish to be able to analyse the critical components that can negatively affect the outcome of the composition.

In our approach, a key ingredient for the aims described above is the compiler BPELtoISPL. BPELtoISPL takes as input an agent's ABs and CCBs in BPEL and produces an ISPL program to be used with the MCMAS model checker [22]. As described below in more detail, the compiler parses the ABs (given in BPEL) to generate a preliminary program enumerating the local states. The CCBs is then parsed to colour the agent's states in terms of compliance (green) or non-compliance (red), as well as basic propositions encoding this. The compiler then adds a number of basic specifications to be checked before prompting the user to add any further properties of interest.

Before presenting the compiler in detail in Section 5, we introduce below the BPEL language, the MCMAS model checker and its input language ISPL. 


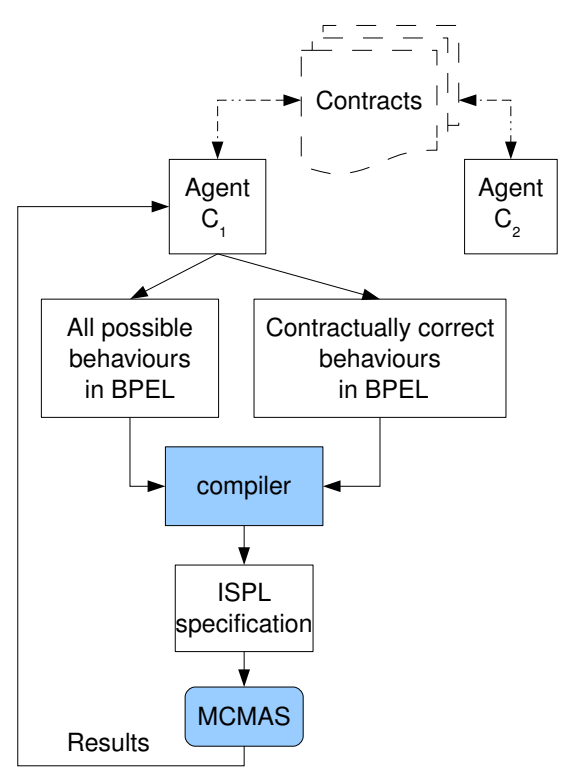

Fig. 2 Verification architecture

\subsection{WSBPEL}

BPEL [10] is a popular and de facto industrial standard for describing service composition, and extends the traditional web services interaction model and provides support for business transactions. Specifically, BPEL is a language for the formal specification of business processes and business interaction protocols. It defines a model and an XML based grammar for the orchestration of business processes.

From an architectural point of view BPEL sits on a layer above the Web Service Description Language (WSDL): permitted operations are stated in WSDL, while their sequencing is expressed in BPEL. BPEL provides the infrastructure for describing the control logic required to coordinate web services participating in a process flow. This is useful for specifying both abstract and executable business processes. An executable process models the behaviour of participants in a specific business interaction, essentially modelling a private workflow. Abstract processes, modelled as business protocols in BPEL, specify the public message exchanges between parties. Business protocols are not executable and do not convey the internal details of a process flow. In other words, executable processes provide the orchestration support, while business protocols focus more on the choreography of the services.

By adopting BPEL the engineer can abstract from the underlying web services implementations; so the business process language effectively becomes a web services API. BPEL utilises WS-Transaction (WS-Coordination) [23] to ensure reliable execution of business processes over multiple workflows, which BPEL logically divides into two distinct aspects. The first is a process description language with support for performing computation, synchronous and asynchronous operation invocations, control-flow patterns, structured error handling, and long-running business transactions. The second is an infrastructure layer that builds on WSDL to capture the relationships between enterprises and processes within a web 
services-based environment. Taken together, these two aspects support the orchestration of web services in a business process, where the infrastructure layer exposes web services to the process layer, which then drives that web services infrastructure as part of its workflow activities. BPEL is normally interpreted and executed by an orchestration engine controlled by one of the participating parties. The engine coordinates the various activities in the process, and compensates the system when errors occur.

The building blocks for a BPEL process are the descriptions of the parties participating in the process, the data that flows through the process and the activities performed during the execution of the process. Some examples of activities include "receive", "reply", "assign", "sequence" and "wait". BPEL also introduces systematic mechanisms for dealing with business exceptions and processing faults. Moreover, BPEL introduces a mechanism to define how individual or composite activities within a unit of work are to be compensated in cases where exceptions occur or a partner requests reversal. We refer the interested reader to [10] for more details.

\subsection{MCMAS and ISPL}

MCMAS [22] is a model checker tailored to the verification of multi-agent systems. MCMAS differs from mainstream model checkers such as NuSMV, SPIN, etc., in that it enables the engineer to verify not only temporal properties but also epistemic, ATL, and other expressive agent-based logics. MCMAS takes as input a MAS model description and a set of specifications to be checked, and it returns the truth value of these on the system provided. MCMAS is fully symbolic checker employing on Ordered Binary Decision Diagrams for efficient representations. Whenever possible, MCMAS produces counterexamples for unsatisfied specifications and witnesses executions for those satisfied. This provides detailed guidance to the engineer in case a revision of the system is required. MCMAS handles fairness efficiently and has been tested on a number of scenarios [22].

The input to MCMAS is given by means of ISPL programs. ISPL (Interpreted Systems Programming Language) is a dedicated agent-based modelling language derived from the formalism of interpreted systems, a popular semantics for the formalisation of MAS [13]. ISPL programs, not dissimilarly to most model checking languages, govern the evolution of agent variables and their interaction by means of Boolean expressions.

MCMAS supports a number of advanced features including variable typing, interactive execution, as well as a range of content-assist input and analysis features in a graphical user interface.

The self-explanatory ISPL program in Table 1 encodes the well-known Train/Controller scenario with one train only and one controller. It can easily be ascertained that ISPL programs uniquely denote interpreted systems as defined above in Section 2.1. Indeed, an agent's instantiation describes its local states (including an optional green and red colouring of its states) as a tuple of its typed variables, its actions, its protocol, and its evolution function. The evaluation function defines satisfaction of basic atoms in view of Boolean combinations of agents' local states. Initial states for the system, any fairness conditions, and specifications to be checked are also defined in an ISPL formula. In this snippet the (satisfied) specification stating that "whenever train 1 is in the tunnel, train 1 knows the light is green" is passed on to MCMAS for verification. 
Table 1 ISPL snippet for the Train/Controller.

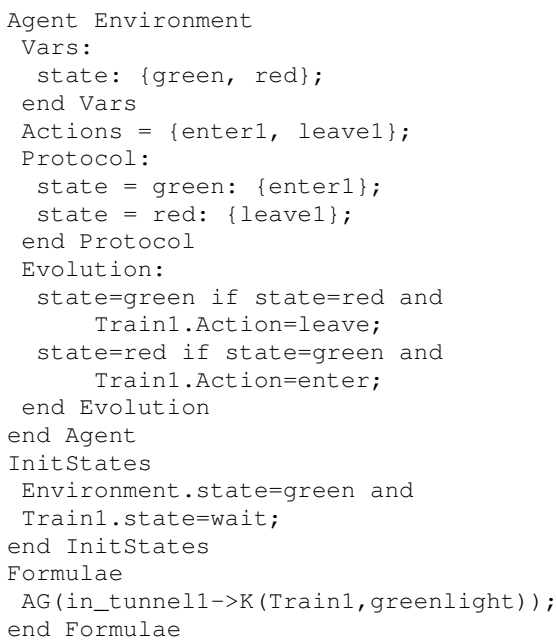

\section{Automatic compilation from WSBPEL into ISPL}

The core component of our framework, shown in Figure 2, is the BPELtoISPL compiler translating a BPEL specification into an ISPL program representing the system to be checked. As we discuss below, in addition to the model generation expressed in ISPL, BPELtoISPL also automatically generates basic atomic propositions and properties to be checked by MCMAS. The internal architecture of the compiler is illustrated in Figure 3 showing a three step construction to generate the corresponding ISPL program from the ABs and CCBs for the system. In a nutshell this is as follows.

1. ABs and CCBs for each BPEL process are read into memory and translated into two corresponding automata. We call contract automaton the automaton representing CCBs for the service in question and behaviour automaton the one representing ABs.

2. We systematically compare the two generated automata. Specifically, starting from the initial state for each state in the behaviour automaton we look for its counterpart in the contract automaton. If this exists, the state is contract compliant, so we label it as green; if not, we label it as red. We proceed with labelling all the states of the behaviour automaton.

3. The resulting labelled behaviour automaton is encoded in ISPL as an agent with red and green states as described in Section 2 as well as transitions corresponding to the BPEL moves. Atoms encoding red states are automatically written in the ISPL output as well as basic specifications. The resulting ISPL file is used by MCMAS for model checking the system.

In what follows we recall the basic automata definitions and discuss the methodology in detail.

5.1 Automata and their semantics

An automaton is a tuple $\left\langle\operatorname{Var}, C, F, S, S_{0}, \Sigma, E\right\rangle$ where 


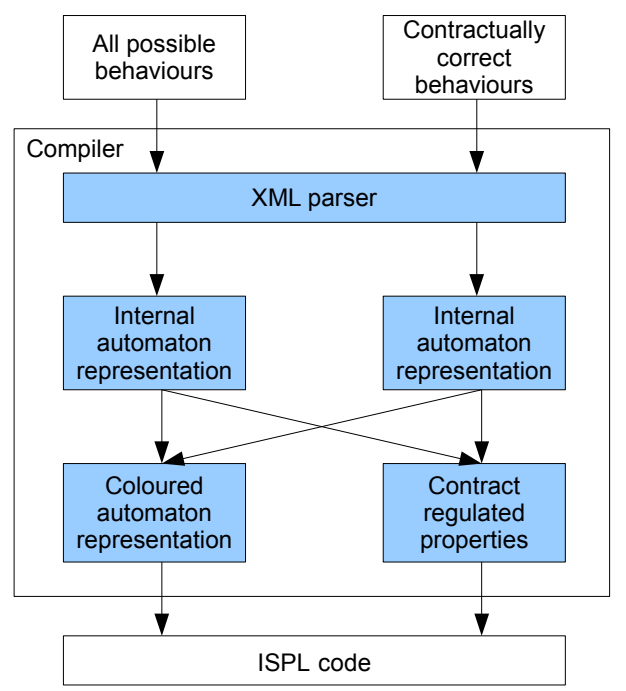

Fig. 3 Internal architecture of the compiler

- Var is a finite set of variables. Each variable $x$ is defined on a domain $R_{x}$;

- $C$ is a finite set of assertions over the set of variables $V a r$;

- $F$ is a finite set of assignments of the form $x:=e x p r$, where $x \in V a r$ and expr is an expression over $\operatorname{Var}$ in the domain of $x$;

- $S$ is a finite set of states;

- $S_{0} \subseteq S$ is the set of initial states;

$-\Sigma$ is a finite set of labels;

- $E$ is the set of transitions over $S \times \Sigma \times C \times 2^{F} \times S$. A transition $e \in E$ can be written as $s \stackrel{\sigma ; c ; f}{\longrightarrow} s^{\prime}$ where $s \in S$ is the source state, $s^{\prime} \in S$ the target state, $\sigma \in \Sigma$ expresses synchronisation conditions with different automata, $c \in C$ is the enabling condition (or the guard) and the postconditions $f \subseteq F$ encode assignments over the variables.

\section{Semantics}

As standard we define automata semantics in terms of their executions. An execution is a possibly infinite sequence of pairs of the form $\left\langle s_{i}, v_{i}\right\rangle, i \in\{0,1, \ldots\}$, where

1. $s_{0} \in S_{0}$,

2. $s_{i}$ is a state from $S$ and $v_{i}$ is an evaluation for the variables in $V a r$.

3. Let $v_{i}(x)$ be the value of $x$ in $v_{i}$. For each adjacent pair $\left\langle s_{i}, v_{i}\right\rangle\left\langle s_{i+1}, v_{i+1}\right\rangle$, the automaton displays a transition $e=s_{i} \stackrel{\sigma ; c ; f}{\longrightarrow} s_{i+1}$, where the assertion $c$ is evaluated as true over $v_{i}$, and for each variable $x \in \operatorname{Var}, v_{i+1}(x)=\operatorname{expr}$ if there is an assignment $(x:=e x p r) \in f$; otherwise, $v_{i+1}(x)=v_{i}(x)$.

A system composed by a number of automata $A_{1}, \ldots, A_{n}$ synchronises by shared actions as follows. For each label $\sigma \in \Sigma=\Sigma_{i} \cup \ldots \cup \Sigma_{n}$ we write $A_{\sigma}=\left\{A_{i} \mid \sigma \in \Sigma_{i}, 1 \leq\right.$ $i \leq n\}$. A transition $e_{i} \in E_{i}$ in $A_{i}$ with label $\sigma$ is executed synchronously if and only if each automaton $A_{j} \in A_{\sigma}(i \neq j)$ executes a transition labelled with $\sigma$. This is formally defined in the product of automata. 


\section{The product of automata}

Let $A_{1}=\left\langle\operatorname{Var}_{1}, C_{1}, F_{1}, S_{1}, S_{0}^{1}, \Sigma_{1}, E_{1}\right\rangle$ and $A_{2}=\left\langle\operatorname{Var}_{2}, C_{2}, F_{2}, S_{2}, S_{0}^{2}, \Sigma_{2}, E_{2}\right\rangle$ be two automata. The product automaton, denoted $A_{1} \| A_{2}$, is the automaton $\left\langle\operatorname{Var}_{1} \cup \operatorname{Var}_{2}, C_{1} \cup\right.$ $\left.C_{2}, F_{1} \cup F_{2}, S_{1} \times S_{2}, S_{0}^{1} \times S_{0}^{2}, \Sigma_{1} \cup \Sigma_{2}, E\right\rangle$. Given a set $f \subseteq F_{i}$ of assignments, let $\operatorname{var}(f) \subseteq$ $V a r_{i}$ be the set of variable being assigned new values by $f$. The set of transitions $E$ is defined as follows.

- For a label $\sigma \in \Sigma_{1} \cap \Sigma_{2}$ and two transitions $e_{1}=s_{1} \stackrel{\sigma ; c_{1} ; f_{1}}{\longrightarrow} s_{1}^{\prime}$ in $E_{1}$ and $e_{2}=$ $s_{2} \stackrel{\sigma ; c_{2} ; f_{2}}{\longrightarrow} s_{2}^{\prime}$ in $E_{2}$ such that $\operatorname{var}\left(f_{1}\right) \cap \operatorname{var}\left(f_{2}\right)=\emptyset$, we have $\left(s_{1}, s_{2}\right) \stackrel{\sigma ; c_{1} \wedge c_{2} ; f_{1} \cup f_{2}}{\longrightarrow}$ $\left(s_{1}^{\prime}, s_{2}^{\prime}\right) \in E$

- For a label $\sigma \in \Sigma_{1} \backslash \Sigma_{2}$ and a transition $s_{1} \stackrel{\sigma ; c_{1} ; f_{1}}{\longrightarrow} s_{1}^{\prime} \in E_{1}$, we have $\left(s_{1}, s_{2}\right) \stackrel{\sigma ; c_{1} ; f_{1}}{\longrightarrow}$ $\left(s_{1}^{\prime}, s_{2}\right) \in E$ for every $s_{2} \in S_{2}$.

- For a label $\sigma \in \Sigma_{2} \backslash \Sigma_{1}$ and a transition $s_{2} \stackrel{\sigma ; c_{2} ; f_{2}}{\longrightarrow} s_{2}^{\prime} \in E_{1}$, we have $\left(s_{1}, s_{2}\right) \stackrel{\sigma ; c_{2} ; f_{2}}{\longrightarrow}$ $\left(s_{1}, s_{2}^{\prime}\right) \in E$ for every $s_{1} \in S_{1}$.

In what follows, we omit labels for simplicity, as well as assignments.

\subsection{Translating BPEL programs into automata}

BPELtoISPL translates BPEL activity constructs into automata transitions associating beginning and end states to the corresponding source and target automaton states recursively. Our translation follows closely the one presented in [24]; we differ from [24] by adding a translation of the BPEL activity "pick". The added translation is in line with the others already presented [24] and warrant no particular discussion (but see the full list presented below). Note that BPEL has no uniformly accepted formal semantics. In addition to [24], other translations have been put forward [25-28]. We chose to follow [24] because it is based on automata and therefore particularly suitable to our aims.

The translator of our realisation uses the following rules.

- A BPEL "assign" activity is composed by a list of assignments. Thus it can be translated as a transition directly with true as its guard. The beginning state of the activity is the source state of the transition and the end state is the target state. An "empty" activity can be seen as a special "assign" activity with no assignments.

- BPEL "receive" or "invoke" activities are translated into transitions in which the beginning state and the end state are the source and the target state of the transition respectively. If an "invoke" activity sends a value expr through the message channel and the corresponding "receive" activity assigns that value to a variable $x$, we consider the assignment in "invoke" to be $y:=\operatorname{expr}$ and the one in "receive" to be $x:=y$, where $y$ is a temporary variable to complete the assignments in "invoke" and "receive". Furthermore we impose that each transition resulting from an "invoke" activity is synchronised (as defined above) to the corresponding transition resulting from the "invoke" activity. The key end result is that the assignment $x:=\operatorname{expr}$ in the corresponding automaton.

- A BPEL "sequence" activity is composed of a sequence of activities $a_{1}, \ldots, a_{n}$. Assume the beginning and end states of each $a_{i}(1 \leq i \leq n)$ are $s_{i}$ and $s_{i}^{\prime}$ respectively. The "sequence" activity merges $s_{i}^{\prime}$ and $s_{i+1}$ into one state for $(1 \leq i \leq n-1)$. The beginning state of the "sequence" is $s_{1}$ and the end state is $s_{n}^{\prime}$. 
- A BPEL "if" activity has a cond logical condition and two execution branches, i.e., the then branch and the else branch. Assume that in the translated automaton the then branch starts from the state $s_{1}$ and finishes at the state $s_{1}^{\prime}$, and, similarly, the else branch starts from $s_{2}$ terminating at $s_{2}^{\prime}$. We translate the "if" activity by generating a new state $s$ and two transitions with empty assignments such that $s \stackrel{\text { cond }}{\longrightarrow} s_{1}$ and $s \stackrel{\neg \text { cond }}{\longrightarrow} s_{2}$, and collapse $s_{2}$ and $s_{2}^{\prime}$ into one state $s^{\prime}$. The beginning and end states are encoded as $s$ and $s^{\prime}$ respectively.

- A BPEL "while" activity is composed of a loop condition cond and a loop body, which we assume is translated into an automaton beginning at state $s_{1}$ and ending at $s_{1}^{\prime}$. To encode the "while" activity we add a new state $s$ preceding the translation of the body, and a new state $s_{2}$ encoding the end state. Then we generate two transitions with empty assignments such that $s \stackrel{\text { cond }}{\longrightarrow} s_{1}$ and $s \stackrel{\text { cond }}{\longrightarrow} s_{2}$, and finally merge $s_{1}^{\prime}$ with $s$.

- A BPEL "pick" activity is composed of a list of "onMessage" activities or "onAlarm" activities. These can be seen as a multi-branch "if" activity. Therefore, similarly to the "if" activity case, we generate a new state $s$ to be the beginning state of the "pick" activity and merge all end states of the "onMessage" or "onAlarm" activities to be the end state of the "pick" activity. Each "onMessage" activity is translated in the same way as the "receive" activities described above. Each "onAlarm" activity is translated into a transition whose guard is the encoding of the activity's triggering condition and whose assignment list is empty.

- BPEL branches of "flow" activities are executed concurrently. To translate them into automata we generate a separate automaton to model each branch as follows. We generate two new states $s_{i}^{0}$ and $s_{i}^{1}$ for branch $i$ and two transitions $e_{i}^{0}=s_{i}^{0} \stackrel{\text { true }}{\longrightarrow} s_{i}$ and $e_{i}^{1}=s_{i}^{\prime} \stackrel{\text { true }}{\longrightarrow} s_{i}^{1}$, where $s_{i}$ is the beginning state of the branch and $s_{i}^{\prime}$ the end state. Additionally we generate three states $s_{0}, s_{1}$ and $s_{2}$ in the main automaton corresponding to the BPEL process with the transitions $e_{0}=s_{0} \stackrel{\text { true; }}{\longrightarrow} s_{1}$, and $e_{1}=s_{1} \stackrel{\text { true }}{\longrightarrow} s_{2}$. The state $s_{0}$ is the beginning state of the "flow" activity and $s_{2}$ is its end state. Furthermore, we synchronise each $e_{i}^{0}$ to $e_{0}$, and each $e_{i}^{1}$ to $e_{1}$. In doing so, we differ from [24], where a "flow" is translated in a way in which all branches are executed sequentially and all possible permutations are represented as a single automaton. The approach used here removes several duplicate transitions, thereby making the translation more efficient.

The automata generated from "if", "while", "pick" and "flow" activities are illustrated in Figure 4.

- BPEL fault handlers and exceptions are translated into transitions as well. To do this we assign a specific value to a variable and add a test in the preceding transition with the guard being the value for the particular variable. Other BPEL handlers are dealt with in the same way. In every state where an exception could happen a copy of the exception/handler transition is produced using this state as its source state (note that these copies have the same target state). While this is correct, observe that doing so would cause one transition to be replicated several times. For efficiency reasons the ISPL code generated is further optimised as we discuss later.

By using the translation above, BPELtoISPL builds the contract automaton from the input BPEL CCBs and the behaviour automaton from the input BPEL ABs.

Once the automata for the BPEL translation have been built following the algorithm above, BPELtoISPL proceeds to colour the behaviour automaton in terms of states of compliance and violation. Once this is done, an ISPL file representing the behaviours and the violation states is produced (see Figure 5). Recall from the previous section that in our initial 

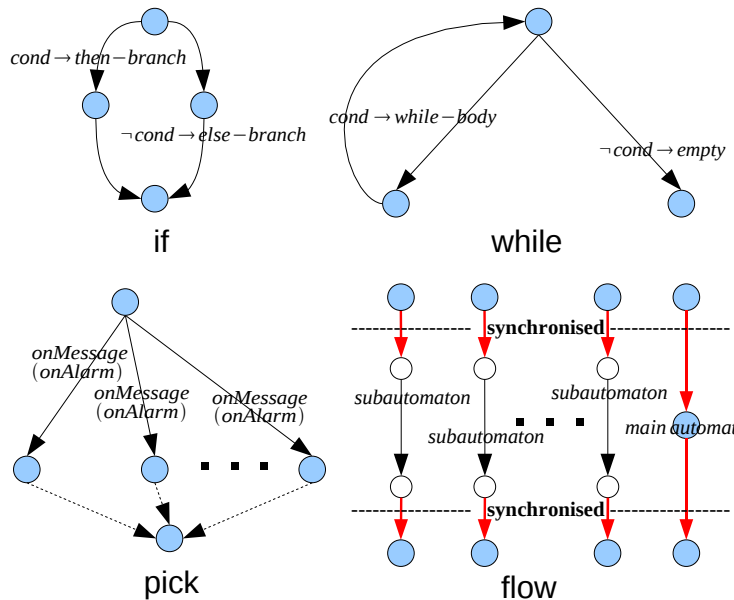

while

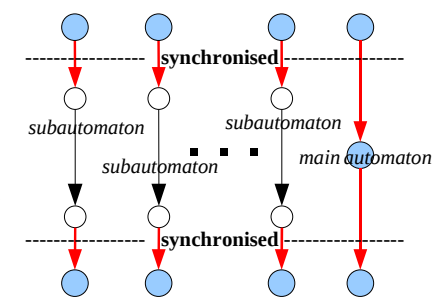

flow

Fig. 4 Translated automaton

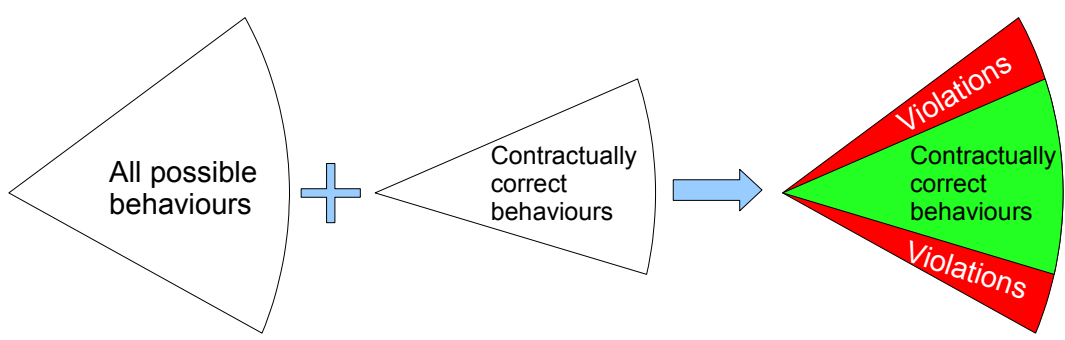

Fig. 5 Generation of ISPL contractually correct behaviours

specification the set of $\mathrm{CCBs}$ is included in all $\mathrm{ABs}$. As a consequence of this, by following the construction above we obtain a contract automaton that is included in the behaviour automaton. Note that the recovery transitions, taking red states to green ones, are also included in the behaviour automaton. More formally, an automaton $A^{\prime}=\left\langle\operatorname{Var}^{\prime}, C^{\prime}, F^{\prime}, S^{\prime}, S_{0}^{\prime}, \Sigma^{\prime}, E^{\prime}\right\rangle$ is included in $A$ if $S_{0}^{\prime}=S_{0}, \operatorname{Var}^{\prime}=\operatorname{Var}, C^{\prime}=C, F^{\prime}=F, \Sigma^{\prime}=\Sigma, S^{\prime} \subseteq S$, and $E^{\prime}=E \backslash E^{\prime \prime}$, where $E^{\prime \prime}$ is the set of transitions whose source state or target state is not in $S^{\prime}$.

Once the contract automaton and the behaviour automaton have been constructed the toolkit proceeds with labelling the states of the behaviour automaton as states of contract compliance (green) or violation (red). To achieve this, BPELtoISPL operates as follows.

1. The initial state of the behaviour automaton is labelled as green.

2. For every transition in the contract automata, the algorithm finds the same transition in the behaviour automata (since each contract automaton is included in a behaviour automaton, this step is guaranteed to succeed) and labels its target state as green.

3. All states left in the behaviour automata that have not been labelled (i.e., that are not green) are labelled as red.

Observe that we do not look for matched states directly because these are named numerically during compilation and therefore the same numbered state in the behaviour automata and the contract automata might correspond to different states. However, transitions get their 
name from the BPEL activities, each of which has a unique name, thereby guaranteeing the correctness of the above. The end result of the translation above are the resulting labelled behaviour automata which can now be translated into ISPL for verification.

\subsection{Generating the ISPL program}

Once the behaviour automata are labelled they can easily be compiled in ISPL for verification (see Section 2). Each automaton is mapped to an agent construct in the ISPL program. Let $\mathcal{A}=\left\{\mathcal{A}_{1}, \ldots, \mathcal{A}_{n}\right\}$ be the set of automata and $A=\{1, \ldots, n\}$ the set of agents. To instantiate an ISPL agent we need to define its basic features, e.g., its set of local states, actions, protocols, evolution function, as well as other system parameters, such as the initial states, propositions, etc. In the following we define the key steps to generate an agent $i \in A$ from an automaton $\mathcal{A}_{i} \in \mathcal{A}$.

1. Local states generation. A local state $l \in L_{i}$ is a valuation for the set of local variables $V a r_{i}$. Thus, the generation of $L_{i}$ is performed through the generation of $\operatorname{Var}_{i}$. If $\mathcal{A}_{i}$ is generated from a BPEL process $p$, then we set

$$
\operatorname{Var}_{i}=\operatorname{Var}_{p} \cup\{\text { state }\},
$$

where $\operatorname{Var}_{p}$ is the set of variables defined in $p$ and state is an additional enumeration variable. Each value of state represents a unique state of $\mathcal{A}_{i}$. If $\mathcal{A}_{i}$ is a "flow" branch in $p$, then we assign

$$
\operatorname{Var}_{i}=\operatorname{Var}_{p}^{\prime} \cup\{\text { state }\}
$$

where $\operatorname{Var}_{p}^{\prime} \subseteq \operatorname{Var}_{p}$ is the set of variables used by $\mathcal{A}_{i}$. In order to reduce the agent's state space, the compiler monitors the usage of every variable $v \in \operatorname{Var}_{p}$. If $v$ is never read by any transitions in $\mathcal{A}_{i}$, then it is discarded from the agent's description in the ISPL file.

2. Local actions generation. Act $t_{i}$ is obtained from the transitions of the corresponding $\mathcal{A}_{i}$. Each transition generates a different action for the agent in question; if two transitions have the same name, they are mapped into the same action.

3. Protocol generation. For any transition $t$ whose source state is represented by $l$ (state), the action to which $t$ is mapped is included in the set of allowed actions in $l$. Obviously, two states $l_{1}, l_{2} \in L_{i}$ have the same set of allowed actions if $l_{1}$ (state $)=l_{2}$ (state).

4. Evolution function generation. Each transition in $\mathcal{A}_{i}$ is translated into an agent evolution item. For a transition $t$ with source state $s_{1}$, target state $s_{2}$, and guard $c$, the evolution item is defined to be of the following form:

$$
\text { state }=s_{2} \text { if state }=s_{1} \text { and } \mathrm{c} \text { and Action }=\mathrm{t} .
$$

This evolution item encodes that if in the current state the variable state has value $s_{1}$ and the guard $c$ is satisfied, the execution of $t$ forces agent $i$ to move to a state where state has value $s_{2}$.

If $t$ is synchronised with another transition $t^{\prime}$ in the automaton $\mathcal{A}_{j} \in \mathcal{A}$, then the evolution item is generated as

$$
\text { state }=s_{2} \text { if state }=s_{1} \text { and } \mathrm{c} \text { and Action=t and } \mathcal{A}_{j} \text {. Action=t'. }
$$

The above forces synchronisation between the agents through the joint action $t, t^{\prime}$ corresponding to the shared actions in the original automata.

If $t$ assigns a value expr to a variable $v$, the assignment is translated by forcing the value to acquire the required value as follows: 
state $=s_{2}$ and $\mathrm{v}=\operatorname{expr}$ if $\cdots$.

If there are multiple copies of $t$, e.g., $t$ represents a fault handler, we use the following form to specify an evolution item for all copies:

$$
\text { state }=s \text { if }\left(\text { state }=s_{1} \text { or state }=s_{2} \text { or } \ldots\right) \text { and } \mathrm{c} \text { and Action }=\mathrm{t} \text { and } \cdots,
$$

where $s_{1}$ and $s_{2}$ are the source states of the $t$ copies and $s$ is their target state. If $t$ is allowed in all states, the above form is simplified as

$$
\text { state }=s \text { if } \mathrm{c} \text { and Action }=\mathrm{t} \text { and } \cdots .
$$

The compiler translates all behaviour automata into an ISPL program as described above. In addition to the agents definitions, ISPL also requires the user to specify the initial states, the atomic propositions and the specifications to be checked. We only describe initial states here and leave the others for the next subsection.

5. Initial states. Since a BPEL process is a sequential program, each process has only one initial state. So every automaton $\mathcal{A}_{i}(1 \leq i \leq n)$ has one initial state $l_{i}^{0}$. The global initial state $s^{0}$ is constructed as $\bigwedge_{i=1}^{n} l_{i}^{0}$. This is written in the ISPL file in the appropriate syntax.

5.4 Automatically generated specifications

In addition to compiling the model, as described above, BPELtoISPL additionally encodes a range of simple specifications to be checked by MCMAS. For each agent $i$ generated in the translation process, the following local atomic propositions are generated by the compiler as follows.

- an atomic local proposition $p_{\text {green }}$, holding on the green states of agent $i$, is defined;

- an atomic proposition $p_{\text {end }}$, holding in the last state of all runs in which the contractual obligations of agent $i$ have been met, is defined;

- an atomic proposition $p_{\text {red }_{i}}$ holding in all red states for agent $i$ is defined.

Observe that while each ISPL agent originates from one and only one BPEL process, a BPEL service may be translated by means of several agents. It is however possible to refer to the contract compliant states of a BPEL process by taking the conjunction of local propositions referring to the green states of the agents the process is mapped to. Formally, we can consider $p_{\text {green }}=\wedge_{i} p_{\text {green }}$ to represent the contract compliant states of BPEL process $p$ where $i$ ranges over the ISPL agents process $p$ was translated to. The toolkit produces these propositions automatically and generates some simple specifications as below. For ease of presentation we assume below every BPEL process is translated into a single agent below (as it happens whenever BPEL "flow" activities are not present); the toolkit handles the general case by means of appropriate conjunctions.

Five basic specifications are generated based on the atomic propositions above. Specifically, for each BPEL process $p$, the toolkit automatically checks the following formulas:

$$
\begin{gathered}
E G p_{\text {green }} \\
E\left(p_{\text {green }} U p_{\text {end }}\right) \\
\text { EF } p_{\text {red }}
\end{gathered}
$$




$$
\begin{aligned}
& A G\left(p_{\text {red }} \rightarrow A F p_{\text {end }}\right) \\
& A G\left(p_{\text {red }} \rightarrow E F p_{\text {end }}\right)
\end{aligned}
$$

The first specification, also discussed in Section 3, expresses the fact that there exists a run in which process $p$ remains always in a state of full compliance with respect to its contracts. Note this specification does not insist on the process actually terminating its run as defined in the BPEL model. Specification (2) refines this concept further by stating that there exists a way for process $p$ to terminate while always remaining in a green state; i.e., there is a way for process $p$ to conduct a contract-compliant run leading to termination of the process. These are properties that we would expect to be satisfied in the composition; if not, we are in the presence of a system in which process $p$ has no way of executing without braking some of the contracts it has adhered to. This provides us with a very coarse validation criteria for the contract regulated composition. Still, this is a feature that can reveal errors either in the BPEL behaviours as programmed, or in the contracts as implemented.

For example, if Specification (1) is satisfied and Property (2) is not, this might mean that the service in question cannot make any progress after some other services violate their contract.

Specification (3) represents a test to check whether an agent may in principle violate its contractual behaviours; Property (4) tests whether the service can always recover from any violation. Note that if Property (3) is not satisfied, then Property (4) will always hold. If both properties are satisfied, we deduce the service may violate some of its contracts but it will always eventually recover from these violations and terminate correctly. Conversely, if Property (3) is satisfied but Property (4) is not, the service is shown not to always recover after some violation of its contracts. A weaker variant of Specification (4) is Specification (5) simply stating that following any violation there is a path leading to recovery and termination. It is instructive to use properties (3,) (4), and (5) in conjunction; e.g, should Specifications (4), (5) be false, we may wish to check the models to establish whether this is indeed a feature of the scenario, or whether we should alter the behaviour of the agent to rectify its exhibited properties.

These are just basic properties pertaining to a single service only. Similar properties can be compiled for the overall system's behaviour considering appropriate conjunctions of the local propositions defined above.

While the compiler generates the properties above automatically for the benefit of the user, for a given system the engineer may wish to explore more complex specifications for the composition. We explore some possibilities in Section 6 while discussing a case study.

\section{Experimental Analysis}

In this section we present a composition of services whose behaviours are regulated by contracts. We also show the verification results obtained by means of the technique above. As stated earlier, we are not concerned with contract negotiation here. Instead we assume all contracts have been negotiated before execution.

\subsection{A Motivating Case Study}

In the scenario we analyse (see Figure 6) the participating contract parties comprise: a principal software provider $(P S P)$, a software provider $(S P)$, a software client $(C)$, an insurance 


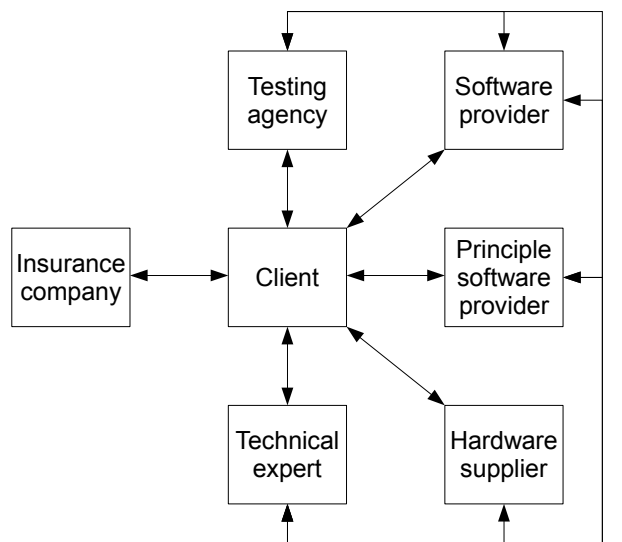

Fig. 6 Interaction (denoted by arrows) between various partners in the composition.

company $(I)$, a testing agency $(T)$, a hardware supplier $(H)$, and a technical expert $(E)$. The high-level workflow of the composition is defined as follows: Client $C$ would like a piece of software developed and deployed on hardware supplied by $H$. To deploy the software, a technical expert $E$ is needed. Components of the software are provided by different software providers. We consider two software providers here: PSP and SP. The components need to be integrated by the providers before the software is delivered to $C$. The software integration is carried out by $P S P$, after $S P$ delivers its component. This happens before any integration has taken place.

Before then $P S P$ and $S P$ twice update each other and $C$ about the progress of the software development. Should the client wish to update the software specification, he can request this at no cost before the second round of updates. Any change required by the client after the second update is considered a violation of the original contract and the client may be charged a penalty at $P S P$ 's discretion. If the penalty is levied, the client can recover from this violation by paying the penalty or by withdrawing the request for changes. If $P S P$ and $S P$ do not send their updates as per schedule, this is also considered a violation and they may be charged a penalty. Every update is followed by a part payment by $C$ to the $P S P$. Payment to $S P$ is handled by $P S P$ and is done once the software is deployed successfully.

After $P S P$ has integrated the components, he sends the integrated software to $T$ for testing. Results from testing are made available to all the parties, i.e., $P S P, S P$, and $C$. If the integration test fails, the components are revised and tested again. Components can be revised twice. If the third test fails, $C$ may cancel the contract with $P S P$. If the testing succeeds, $C$ invokes $I$ to get the software insured. $C$ then invokes $H$ to order the hardware. Finally $C$ invokes $E$ to get the software deployed. If the software cannot be deployed then the hardware and the components have to be re-evaluated. Software components can be revised twice at no penalty. If the third test fails $C$ cancels the contract with $P S P$ and $H$. Figure 7 summarises the obligations of the $P S P$ and $C$. Figure 8 illustrates informally some of the conditions under which some local violations may occur.

From the above scenario it can be seen that a notion of correctness for each party can be deduced from the contracts applicable to them. Any deviation from the behaviour identified in the contract is considered a violation. The contract also specifies mechanisms for recover- 
$P S P$ 's obligations:

1. Update $S P$ and $C$ twice about the progress of the software.

2. Integrate the components and send them to $T$ for testing.

3. If components fail, revise the software, integrate the revised software and send them for testing.

4. Make payment to $S P$ after successful deployment of software.

$C$ 's obligations:

1. Refrain to request any changes after the second round of updates.

2. Pay penalty if changes are requested after second round of updates.

3. Make payment to the $P S P$ after every update.

Fig. 7 Obligations of Contract parties.

\begin{tabular}{|c|c|c|c|}
\hline & Agent & Violation condition & Recovery \\
\hline 1 & $P S P$ & $\begin{array}{l}\text { - fails to send messages to } S P \text { and/or } C \text { in } \\
\text { the first and/or second run of update. }\end{array}$ & pay penalty charge \\
\hline 2 & & - fails to send payment to $S P$. & N/A \\
\hline 3 & $S P$ & $\begin{array}{l}\text { - fails to send update messages to } P S P \text { or } \\
C \text {. }\end{array}$ & pay penalty charge \\
\hline 4 & & - fails to send its components to $P S P$. & N/A \\
\hline 5 & $C$ & - request changes after second update. & pay penalty charge or withdraw changes \\
\hline 6 & & - fails to send the payment to $P S P$. & N/A \\
\hline 7 & $T$ & $\begin{array}{l}\text { - fails to send the testing report to } C \text {, } \\
P S P \text { and/or } S P \text {. }\end{array}$ & N/A \\
\hline 8 & $H$ & - fails to deliver the hardware system to $C$. & N/A \\
\hline 9 & & - ignores the deployment failure. & N/A \\
\hline 10 & $E$ & $\begin{array}{l}\text { - fails to deploy the software on the hard- } \\
\text { ware system. }\end{array}$ & N/A \\
\hline 11 & $I$ & - fails to process the claim of $C$. & N/A \\
\hline
\end{tabular}

Fig. 8 Agents and their violation conditions.

ing from violations. While the scenario may seem artificially constructed, it is actually taken almost verbatim from a software procurement workflow of a leading IT industry.

\subsection{Basic model}

We evaluated our compilation and verification mechanism on the case study above. We represented the composition in terms of a BPEL orchestration. The following BPEL code represents the full behaviour of the client $C$, when receiving updates from $P S P$ and $S P$. Note that for brevity, only essential information is shown. The BPEL-contract is the same as BPEL-behaviour except that it defines only contractually correct and therefore limited behaviours.

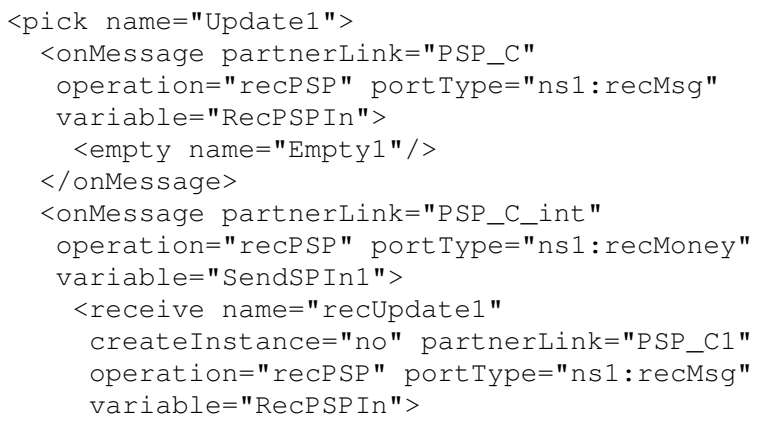




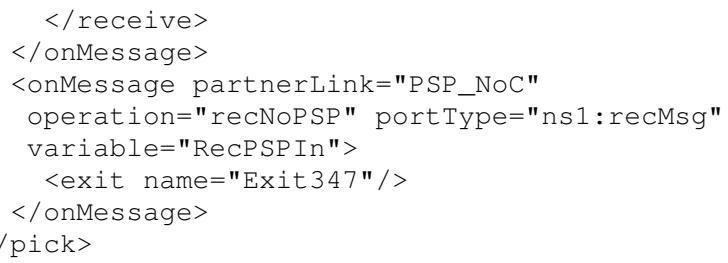

The translation generates the following ISPL program for the client.

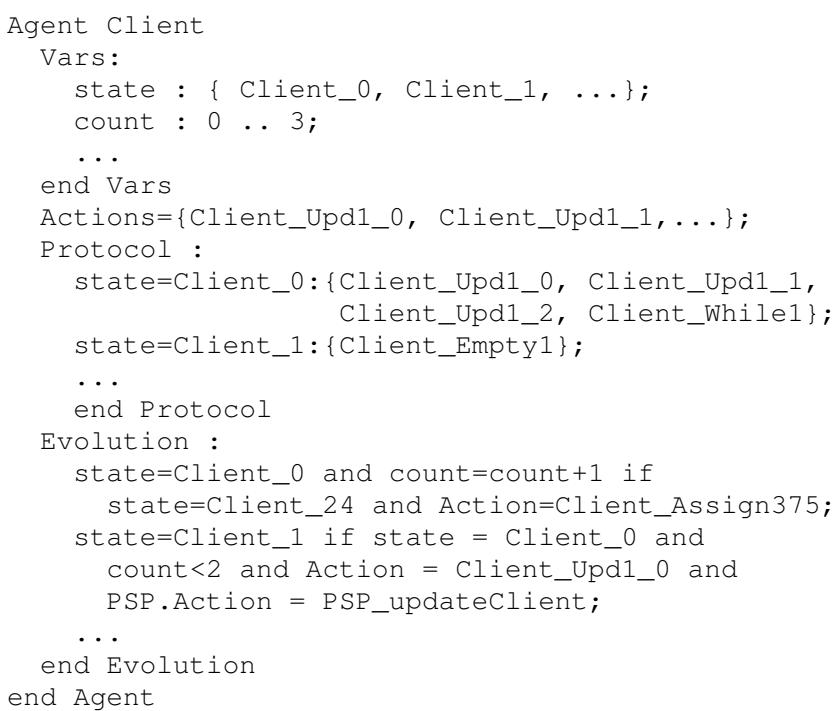

The following listing gives an example about how to define atomic propositions and properties in ISPL.

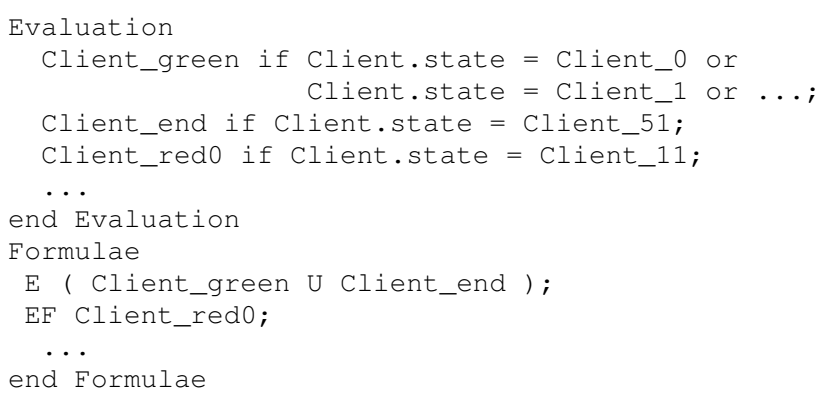

In addition to the basic properties automatically generated by the compiler, we manually added a few more complex properties to the model. Those properties were also studied in $[11,12]$. Some atomic propositions, e.g., "receiveSoftware" and "softwareTested", were also added to the ISPL code manually to test further specifications as we describe below.

\subsection{Further specifications}

In this subsection we formalise various properties of compliance (or lack of) for the motivating case study outlined in Section 6.1 using the syntax defined in Section 2. 
- Whenever PSP is in a green state (i.e., is in a state of compliance), he knows the contract can be eventually fulfilled successfully. We encode this as:

$$
A G\left(P S P_{-} \text {green } \rightarrow K_{P S P} E F\left(P S P \_ \text {end }\right)\right)
$$

Intuitively this property should not hold in the example above because even though $P S P$ remains in compliance, the software might either fail to pass the testing phase or not be deployed. Conversely the following two specifications should be satisfied.

- There exists a path where $C$ is always in compliance with the contract until he eventually receives the software.

$$
E\left(C_{-} \text {green } U \text { receiveSoftware }\right)
$$

- In some of the paths where $P S P$ and $S P$ are always in compliance, the software can be eventually integrated and tested.

$$
E\left(\left(P S P_{-} \text {green } \wedge S P_{-} \text {green }\right) U(\text { softwareIntegrated } \wedge \text { EFsoftwareTested })\right)
$$

- PSP knows that it is possible that PSP, SP, C, I, H, T and $E$ are all in compliance until the software is delivered.

$$
K_{P S P} E(\text { all_green } U \text { softwareDelivered }),
$$

where $a l l_{-}$green represents $P S P_{-}$green $\wedge S P_{-}$green $\wedge C_{-}$green $\wedge T$ Green $\wedge H_{-}$green $\wedge$ $E_{-}$green $\wedge I_{-}$green. While it would be reasonable for this specification to hold in the scenario, in the BPEL we analysed this specification resulted false because of a possible livelock of the testing agent. This is potentially a finding that would lead to a revision in the implementation.

- There is a trace in which the client is always in contract compliant states until the software is delivered (while the client remains compliant) before the client enters a violation.

$$
E\left(C_{-} \text {green } U E\left(\left(C_{-} \text {green } \wedge \text { softwareDeployed }\right) U \neg C_{-} \text {green }\right)\right)
$$

This specification is satisfied in the BPEL code tested because the client may not honour the final payment.

- It is possible that $S P$ is always in compliance before failing to provide the component requested by the $P S P$ forever.

$$
E\left(S P \_ \text {green } U E G\left(\neg S P \_ \text {green } \wedge \text { componentNotProvided }\right)\right)
$$

The following two specifications are satisfied in the scenario.

- PSP might not send the first update to $C$ as per schedule and might only send it after paying a penalty to $C$. However from then on, $P S P$ may always in compliance.

$$
\begin{aligned}
E\left(P S P _ { - } \text { greenU } \left(\neg P S P_{-} \text {green } \wedge\right.\right. & \text { noFirstUpdate } \wedge \\
& E X(\text { payPenalty } \wedge \text { EGPSP_green })))
\end{aligned}
$$

- It is possible that $C$ withdraws the request for a change made after the second update.

$$
\text { EF } E\left(\left(\neg C_{-} \text {green } \wedge \text { illegalRequest }\right) U\left(C_{-} \text {green } \wedge \text { withdrawRequest }\right)\right)
$$


The generated ISPL model was encoded automatically by MCMAS by using 134 BDD variables: 49 BDD variables for local states (the same number of BDD variables are constructed for the transition relation) and 36 for local actions. The total number of global states is approximately $10^{6}$. On a machine running Fedora 13 (kernel 2.6.36.6-147-fc13) on an Intel Core 2 Duo E8400 3.0GHz with 4GB memory it took about 8 seconds for MCMAS to verify 44 properties including the specifications discussed above. The memory consumption was approximately $12 \mathrm{MB}$. The code for the scenario as translated is included in the MCMAS release [29].

In this example the basic properties expressed by formulas (1)-(3) in Section 5 were shown to hold on the model for all agents in the system. Formula (4) does not hold for any. As presented in Figure 8, Formula (5) holds for some agents (i.e., $P S P, S P$, and $C$ ) but not for all of them. This means not only all parties can fulfil their contractual obligations successfully, but also that all the violations shown in Figure 8 are potentially realised in the model. In some cases there is no recovery from a violation. The toolkit also returned results for the properties discussed earlier in this section in line with our intuitions. Table 2 summarises some of the findings.

We do not compare experimental results with other verification approaches to BPEL, such as those described in the next section. Firstly, we are not aware of other work supporting temporal-epistemic properties in a contract-oriented setting. Secondly, as far as we know, no other similar compiler has been released as open-source, thereby making any comparison difficult even for the CTL fragment. Notwithstanding the above MCMAS uses the de-facto leading BDD library so we would not expect significant improvements in the verification time by using other BDD-based solutions.

Table 2 Verification of the properties.

\begin{tabular}{|c|c|c|c|}
\hline Property & Satisfaction & Property & Satisfaction \\
\hline 6 & no & 10 & yes \\
\hline 7 & yes & 11 & yes \\
\hline 8 & yes & 12 & yes \\
\hline 9 & no & 13 & yes \\
\hline
\end{tabular}

\section{Related work and conclusions}

Several deontic formalisms have been proposed in computer science over the past twenty years; see, e.g., [30] for a first collection. A number of logics, including [31], have been put forward to distinguish between ideal (correct, acceptable) and actual (possibly incorrect) behaviour in the context of computing systems. The formalism used in this paper belongs to this line of work. Specifically, the formal model used here, i.e., temporal deontic systems of Section 2, is an extension to branching time of deontic interpreted systems [14,17], a formalism for reasoning about knowledge and correctness. The presentation given here follows that of [32], where a bounded model checking technique was also given.

The notion of compliance has acquired considerable attention in the past few years in the area of agents and generally in AI. For example, [33] presents $n \mathrm{C}+$, an extension of $\mathrm{C}+$, the action language proposed by Giunchiglia et al., to deal with persistence [34], where acceptable and disallowed transitions are specified. Differently from the work reported here, 
the emphasis in [33] is on labelling transitions rather than states; there is also more attention on the modelling, whereas our concerns are primarily related to efficient verification. More recently, [35] presented a formalism whereby a normative system is represented by a subset of a transition system representing a system's behaviours, also in terms of allowed and disallowed transitions. However, differently from the present contribution, the authors are concerned with the meta-logical properties of the logic and the theoretical complexity of the resulting model checking problem. In constrast to these, here we take a relatively wellunderstood formalism and pursue effective verification of contract-regulated web services.

The above are only two lines of the many directions of theoretical work currently being investigated. Given the emphasis of the present work does not concern the underpinning formal models, we refer the reader interested in the former to the proceedings of the many workshops and thematic symposia regularly held on these topics. Instead, below we discuss some of the more prominent contributions in verification of web services, focusing on those that are more closely related to the approach taken here.

Solaiman et al. [36] discuss an approach based on specifying contracts as finite state machines (FSM). The rights and obligations of the contractual parties are extracted from the clauses of the contracts and mapped into the states, transitions, and output functions of a FSM. The contracts are verified using the model checker SPIN [37]. Using this methodology only LTL properties can be verified. While this work is relevant, it focuses on the contract themselves, not on the agents' behaviours in the presence of contracts that may be broken.

Foster et al. [25] discuss a model based approach to verifying web service composition based on workflows. The technique is based on establishing trace equivalence between the design and implementation. The workflow specifications are modelled as message sequence charts in UML and then abstracted into the FSP process algebra, while the implementation is mechanically translated to FSP. The LTSA tool is used for analysing the resulting implementation.

Pistore et al. [5] present a technique based on the known approach to planning via model checking. Specifically the paper focuses on a general framework for planning under uncertainty applied to the automated composition and monitoring of BPEL processes. The approach has been devised to deal with non-deterministic domains, partial observability and extended goals. Somewhat in the same spirit Lazovik et al. [38] present a planning framework for services specified as BPEL processes based on the principle of interleaved planning and execution. The execution of planned goals is monitored against predefined standard business processes and interacts with the user to achieve satisfaction of the goal. User requests are specified in XSRL, a high level representation format.

Hull et al. [39] discuss how Mealy signatures may facilitate the verification of service properties of e-compositions specified in BPEL. Propositional Linear Temporal Logic is used for specifying properties. Verification results were given in the context of bounded queues, unbounded queues and "white box" mediators. However, while a bounded queue composition can be simulated by a finite state automaton, it has been argued that Mealy machines composed using unbounded queues may lead to undecidability in the LTL verification problem.

Walton [40] defines interactions between Web services in the form of a dialogue protocol. A dialogue protocol encompasses patterns of the message exchange and rules of the dialogue. A Multi-Agent Protocol language was used for expressing the dialogue protocol and the SPIN Model checker [37] was used to perform verification.

Fu et al. [24] suggested modelled services as peers interacting via asynchronous messages in XML format. The authors present a compiler translating the conversation protocol to PROMELA and show that properties of conversation protocols, expressed in LTL, can be 
automatically verified using SPIN. Our translation into automata is based on the one presented originally in this paper. Other translations from BPEL rely on different semantics; for example [28] uses the $\pi$ calculus while [27] is based on Petri nets.

Although not based on BPEL, Huang et al. [41] present an approach to verifying OWL-S process models by using the test cases generated in the model checking process. The authors extend the BLAST model checker [42] to handle concurrency constructs in OWL-S and propose enhancements in OWL-S and PDDL to facilitate automated test case generation.

Baresi et al. [26] explored the use of the Bogor model checker [43] to verify BPEL workflows. At design time a composition of BPEL processes is modelled using the ALBERT assertion language, which is then translated into Bogor's input language for model checking. At run time the assertions defined in ALBERT are checked to monitor the required properties. Mongiell et al. [44] propose a formal model to verify executions of BPEL processes. Related to some of the work we presented here, they also report on a compiler from BPEL into the input language of the NuSMV model checker [45] in order to check CTL properties on the models for BPEL compositions. Similarly, Kazhamiakin et al. [46] also adopted NuSMV and SPIN to verify web service compositions, but they focused on the communication models in the composition, especially asynchronous and buffered communications. To do so, they developed a parametric model to describe a hierarchy of communications and presented an algorithm to build the most adequate model to capture behaviours related to communication.

Several tools other than model checkers have been employed for the verification of web service compositions. For example, Rouached et al. [47] use event calculus [48] together with one of the available engines to model and verify BPEL processes. In addition to the properties specified in the event calculus, the architecture proposed can also check nonfunctional constraints on the behaviours of individual service providers. Schlingloff et al. [49] model BPEL processes in Petri nets to perform usability analysis and check ATL [50] properties. Related to this, more recently Ouyang et al. [51] give a very detailed translation of BPEL into Petri nets, but their approach focus on reachability analysis, check conflicting message-consuming activities, and verify garbage collection on queued messages.

Within the domain of MAS [52] addresses the problem of reasoning about the correctness of business contracts using commitments. The constraints considered as part of the contract specifications do not include temporal nor epistemic modalities. Baldoni et al. [53] propose a method to verify the conformance of agent behaviours with respect to a public protocol. Their focus is on the interoperability among agents not on the obligations and violations as we addressed here. Similarly to [52] they also do not consider CTL and epistemic properties.

The above is only a quick summary of some of the many valuable contributions in the rich literature on verification of web service compositions. However, none of the works in the literature address the issue of violation, non-compliance, and recovery of contract-based behaviours in a web service setting. Still, notions such as SLAs, protocols, and contracts appear central in the context of multi-agent systems, where entities are autonomously maximising their own objectives possibly leading to non-compliant behaviour. Similarly, while the MAS community has made inroads into verifying multi-agent systems by means of rich intensional logics such as epistemic, deontic and ATL logics, these have typically not been considered yet in the web service literature.

In summary, our contribution focuses on a novel technique for the verification of contractregulated service compositions. In our approach services and contracts are specified as BPEL behaviours. These descriptions are semi-automatically compiled into ISPL, and then verified using the symbolic model checker MCMAS. We find the approach has three salient 
features distinguishing it from the literature. First, we support a rich and expressive range of specifications coming directly from multi-agent systems theories; we find these particularly appropriate in the context of sophisticate web service interactions. Second, we can reason both in terms of contract-compliant and violations, as well as more sophisticated concepts including various notions of recovery. Third, the approach is essentially automatic and paired with a state-of-the-art model checker for multi-agent systems, thereby enabling the possibility of verifying very large state spaces such as those arising from real scenarios.

As part of future work, we highlight some of the objectives which we hope to achieve and incorporate in the framework presented:

- As stated in the earlier parts of the paper the formalism of deontic interpreted systems in its coarse red/green partitioning of local states cannot support reasoning about multiple local violations as required, for example, in contrary-to-duty situations or multiple contract clauses. Extensions of this work could include further labelling to account for these needs.

- Since MCMAS does not support real-time systems, some BPEL constructs such as deadline and timeout are currently translated into non-deterministic behaviours. For real-time properties, a secondary model checker, such as UPPAAL [54] or Verics[55], can be integrated into the framework.

- Currently the transformation of contracts from an informal representation to BPEL is a manual process. Recently several frameworks with dedicated contracting languages, e.g., [21], have been proposed in order to compile electronic contracts from their informal representations. No language proposed so far seamlessly integrates behaviour descriptions with contracts, however this would be something worth studying further.

Acknowledgements: The research described in this paper was supported by the European Commission Framework 6 funded project CONTRACT (IST Project Number 034418). The authors would like to thank the anonymous referees for valuable comments on the paper.

\section{References}

1. Wooldridge, M.: An introduction to multi-agent systems. John Wiley, England (2002)

2. D Booth, H Haas, F McCabe, E Newcomer, M Champion, C Ferris and D Orchard: Web service architecture. W3c working group note 11 february 2004 (2004). http://www.w3.org/TR/ws-arch/

3. Lamanna, D.D., Skene, J., Emmerich, W.: Slang: A language for defining service level agreements. In: FTDCS, pp. 100- (2003)

4. Morgan, G., Parkin, S., Molina-Jimenez, C., Skene, J.: Monitoring middleware for service level agreements in heterogeneous environments. Challenges of Expanding Internet: E-Commerce, E-Business, and E-Government (2006)

5. Pistore, M., Barbon, F., Bertoli, P., Shaparau, D., Traverso, P.: Planning and monitoring web service composition. In: AIMSA, pp. 106-115 (2004)

6. X. Fu T. Bultan and J. Su: Conversation Protocols: A Formalism for Specification and Verification of Reactive Electronic Services. In: CIAA, vol. LNCS 2759, pp. 188-200. Springer-Verlag (2003)

7. Penczek, W., Lomuscio, A.: Verifying epistemic properties of multi-agent systems via bounded model checking. Fundamenta Informaticae 55(2), 167-185 (2003)

8. Bordini, R., Fisher, M., Pardavila, C., Visser, W., Wooldridge, M.: Model checking multi-agent programs with CASP. In: CAV'03, vol. LNCS 2725, pp. 110-113. Springer-Verlag (2003)

9. Wooldridge, M., Huget, M.P., Fisher, M., Parsons, S.: Model checking for multiagent systems: the mable language and its applications. International Journal on Artificial Intelligence Tools 15(2), 195-226 (2006)

10. OASIS Web service Business Process Execution Language (WSBPEL) TC: Web service Business Process Execution Language Version 2.0 (2007) 
11. A. Lomuscio and H. Qu and M. Solanki: Towards verifying compliance in agent-based web service compositions. In: Proceedings of The Seventh International Joint Conference on Autonomous Agents and Multi-agent systems (AAMAS-08), pp. 265-272. ACM Press (2008)

12. Lomusico, A., Qu, H., Solanki, M.: Towards verifying contract regulated service composition. In: Proceedings of IEEE International Conference on Web Services (ICWS 2008), pp. 254-261. IEEE Computer Society (2008)

13. Fagin, R., Halpern, J.Y., Moses, Y., Vardi, M.Y.: Reasoning about Knowledge. MIT Press, Cambridge (1995)

14. Lomuscio, A., Sergot, M.: Deontic interpreted systems. Studia Logica 75(1), 63-92 (2003)

15. Lomuscio, A., Woźna, B.: A complete and decidable axiomatisation for deontic interpreted systems. In: Proceedings of the 8th International Workshop on Deontic Logic in Computer Science (DEON'06), vol. 4048, pp. 238-254. Springer-Verlag (2006)

16. Clarke, E.M., Grumberg, O., Peled, D.A.: Model Checking. The MIT Press, Cambridge, Massachusetts (1999)

17. Lomuscio, A., Sergot, M.: A formalisation of violation, error recovery, and enforcement in the bit transmission problem. Journal of Applied Logic 2(1), 93-116 (2004)

18. Anderson, A.R.: A reduction of deontic logic to alethic modal logic. Mind 58, 100-103 (1958)

19. Kanger, S.: New foundations for ethical theory. In: R. Hilpinen (ed.) Deontic Logic: Introductory and Systematic Readings, pp. 36-58. Reidel Publishing Company (1971)

20. Prisacariu, C., Schneider, G.: A formal language for electronic contracts. In: Proceedings of FMOODS07, LNCS, vol. 4468, pp. 174-189. Springer (2007)

21. Panagiotidi, S., Vazquez-Salceda, J., Alvarez-Napagao, S., Ortega-Martorell, S., Willmott, S., R. Confalonieri, P.S.: Contracting agent language. In: Symposium on Behaviour Regulation in Multi-Agent Systems (2008)

22. Lomuscio, A., Qu, H., Raimondi, F.: MCMAS: A model checker for the verification of multi-agent systems. In: Proceedings of CAV 2009, LNCS, vol. 5643, pp. 682-688. Springer (2009)

23. OASIS Web Services Transaction (WS-TX) TC: Web services coordination (ws-coordination) version $1.2(2009)$

24. Fu, X., Bultan, T., Su, J.: Analysis of interacting BPEL web services. In: 13th international conference on World Wide Web, pp. 621-630. ACM Press (2004). DOI http://doi.acm.org/10.1145/988672.988756

25. Foster, H., Uchitel, S., Magee, J., Kramer, J.: Model-based verification of web service compositions. In: Proceedings of the 10th IEEE International Conference on Automated Software Engineering. IEEE Press (2003)

26. Baresi, L., Bianculli, D., Ghezzi, C., Guinea, S., Spoletini, P.: Validation of web service compositions IET Softw. 1(6), 219-232 (2007)

27. Ouyang, C., Verbeek, E., van der Aalst, W.M.P., Breutel, S., Dumas, M., ter Hofstede, A.H.M.: Formal semantics and analysis of control flow in ws-bpel. Science of Computer Programming 67(2-3), 162-198 (2007)

28. R, L., Mazzara, M.: A pi-calculus based semantics for ws-bpel. Journal of Logic and Algebraic Programming 70(1), 96-118 (2007)

29. Lomuscio, A., Qu, H., Raimondi, F.: Mcmas 0.9 beta. http://www-lai.doc.ic.ac.uk/mcmas (2009)

30. Meyer, J.J.C., Wieringa, R.J. (eds.): Deontic Logic in Computer Science: Normative System Specification. John Wiley \& Sons (1993)

31. Jones, A.J.I., Sergot, M.J.: On the characterisation of law and computer systems: The normative systems perspective. In: Deontic Logic in Computer Science: Normative System Specification, chap. 12. Wiley (1993)

32. Wozna, B., Lomuscio, A., Penczek, W.: Bounded model checking for deontic interpreted systems. Electronic Notes in Theoretical Computer Science 126, 93-114 (2005)

33. Craven, R., Sergot, M.J.: Agent strands in the action language nc+. J. Applied Logic 6(2), 172-191 (2008)

34. Giunchiglia, E., Lee, J., Lifschitz, V., McCain, N., Turner, H.: Nonmonotonic causal theories. Artif. Intell. 153(1-2), 49-104 (2004)

35. Ågotnes, T., Hoek, W.v., Rodríguez-Aguilar, J.A., Sierra, C., Wooldridge, M.: On the logic of normative systems. In: M.M. Veloso (ed.) IJCAI, pp. 1175-1180 (2007)

36. Solaiman, E., Molina-Jiménez, C., Shrivastava, S.K.: Model checking correctness properties of electronic contracts. In: ICSOC (2003)

37. Holzmann, G.J.: SPIN Model Checker, The: Primer and Reference Manual. Addison Wesley Professional $(2003)$ 
38. Lazovik, A., Aiello, M., Papazoglou, M.: Associating assertions with business processes and monitoring their execution. In: ICSOC '04: Proceedings of the 2nd international conference on Service oriented computing, pp. 94-104. ACM Press, New York, NY, USA (2004). DOI http://doi.acm.org/10.1145/ 1035167.1035182

39. Hull, R., Benedikt, M., Christophides, V., Su, J.: E-services: a look behind the curtain. In: Proceedings of the twenty-second ACM SIGMOD-SIGACT-SIGART symposium on Principles of database systems, pp. 1-14. ACM Press (2003). DOI http://doi.acm.org/10.1145/773153.773154

40. Walton, C.D.: Model - checking multi-agent web services. In: AAAI 2004: Spring Symposium on Semantic Web Services (2004)

41. Huang, H., Tsai, W., Paul, R., Chen, Y.: Automated model checking and testing for composite web services. In: Proc. of ISORC'05, pp. 300-307. IEEE Computer Society (2005). DOI http://dx.doi.org/ 10.1109/ISORC.2005.16

42. Henzinger, T.A., Jhala, R., Majumdar, R., , Sutre, G.: Software verification with Blast. In: Proceedings of the Tenth International Workshop on Model Checking of Software (SPIN), pp. 235-239. Lecture Notes in Computer Science 2648, Springer-Verlag (2003). URL http: / /www.gigascale.org/pubs / 399. html

43. Robby, Dwyer, M.B., Hatcliff, J.: Bogor: A flexible framework for creating software model checkers. In: TAIC PART, pp. 3-22. IEEE Computer Society (2006)

44. Mongiello, M., Castelluccia, D.: Modelling and verification of bpel business processes. In: the 4th Workshop on Model-Based Development of Computer-Based Systems and 3rd International Workshop on Model-Based Methodologies for Pervasive and Embedded Software, pp. 144 - 148. IEEE Computer Society (2006)

45. Cimatti, A., Clarke, E.M., Giunchiglia, E., Giunchiglia, F., Pistore, M., Roveri, M., Sebastiani, R., Tacchella, A.: NUSMV2: An open-source tool for symbolic model checking. In: Proceedings of the 14th International Conference on Computer Aided Verification (CAV'02), LNCS, vol. 2404, pp. 359-364. Springer-Verlag (2002)

46. Kazhamiakin, R., Pistore, M., Santuari, L.: Analysis of communication models in web service compositions. In: WWW, pp. 267-276. ACM (2006)

47. Rouached, M., Perrin, O., Godart, C.: Towards formal verification of web service composition. In: the 4th International Conference on Business Process Management (BPM06, LNCS, vol. 4102, pp. 257-273. Springer-Verlag (2006)

48. Kowalski, R., Sergot, M.: A logic-based calculus of events. New Generation Computing 4(1), 67 - 95 (1986)

49. Schlingloff, H., Martens, A., Schmidt, K.: Modeling and model checking web services. Electr. Notes Theor. Comput. Sci. 126, 3-26 (2005)

50. Alur, R., Henzinger, T.A., Kupferman, O.: Alternating-time temporal logic. Journal of the ACM 49(5), 672-713 (2002)

51. Ouyang, C., Verbeek, E., van der Aalst, W.M.P., Breutel, S., Dumas, M., ter Hofstede, A.H.M.: Formal semantics and analysis of control flow in ws-bpel. Science of Computer Programming 67(2-3), 162-198 (2007)

52. Desai, N., Narendra, N.C., Singh, M.P.: Checking correctness of business contracts via commitments. In: AAMAS '08: Proceedings of the 7th international joint conference on Autonomous agents and multiagent systems, pp. 787-794. International Foundation for Autonomous Agents and Multiagent Systems, Richland, SC (2008)

53. Baldoni, M., Baroglio, C., Martelli, A., Patti, V.: Verification of protocol conformance and agent interoperability. In: CLIMA VI, LNCS, vol. 3900, pp. 265-283. Springer (2005)

54. Bengtsson, J., Larsen, K., Larsson, F., Pettersson, P., Yi, W., Weise, C.: New generation of UPPAAL. In: Proceedings of the International Workshop on Software Tools for Technology Transfer (1998)

55. Dembiński, P., Janowska, A., Janowski, P., Penczek, W., Pólrola, A., Szreter, M., Woźna, B., Zbrzezny, A.: VerICS: A tool for verifying Timed Automata and Estelle specifications. In: Proc. of the 9th Int. Conf. on Tools and Algorithms for the Construction and Analysis of Systems (TACAS'03), LNCS, vol. 2619, pp. 278-283. Springer-Verlag (2003) 